\title{
Off-shell nilpotent (anti-)BRST symmetries for a free particle system on a toric geometry: superfield formalism
}

\author{
R. Kumar \\ S. N. Bose National Centre for Basic Sciences, \\ Block JD, Sector III, Salt Lake, Kolkata-700098, India \\ E-mails: rohit.kumar@bose.res.in; raviphynuc@gmail.com
}

\begin{abstract}
We derive the off-shell nilpotent as well as absolutely anticommuting (anti-)BRST symmetry transformations, within the framework of superfield approach to BRST formalism, for a free particle system constrained to move on a torus. We also construct the most appropriate gauge-fixed Lagrangian which respects the (anti-)BRST symmetry transformations.
\end{abstract}

PACS numbers: 11.10.Ef, 11.15.-q

Keywords: Dirac brackets; superfield formalism; (anti-)BRST symmetries 


\section{Introduction}

The mathematical properties associated with the toric geometry have raised a great deal of interest of the theoretical physicists in the context of (super) string and gauge theories [1, 2]. For instance, the geometrical structure of a closed string is one of the simplest examples of the torus. The AharonovBohm and Casimir effects have also been studied in the realm of noncommutative toric geometry [3, 4]. The geometrical structure and physical characteristics of the charged torus has been studied (see, e.g., [5] for details). Moreover, the square-root quantization of elementary particle masses and charges on the torus is also carried out [6, 7].

The Becchi-Rouet-Stora-Tyutin (BRST) formalism is one of most elegant methods to quantize the gauge theories [8, 9, 10, 11]. In this formalism, the unitarity and the quantum "gauge" (i.e. BRST and anti-BRST) symmetries are respected together at any arbitrary order of perturbative computations. Recently, the BRST symmetries for a free particle system on a toric geometry have been discussed and its quantization has also been carried out in [12] in the context of Batalin-Fradkin-Vilkovisk formalism [13, 14].

The key properties (i.e. nilpotency and absolute anticommutativity) associated with the (anti-)BRST symmetry transformations have their geometric origin in the superfield formalism and these properties become transparent [15, 16]. Within the framework of superfield approach to BRST formalism, the model of rigid rotor (see, e.g., [17]) has been studied where a specific choice for the gauge potentials has been chosen to derive the proper (anti-) BRST symmetries [18. Furthermore, the model of rigid rotor provides a toy model for the Hodge theory where the continuous symmetries of the model provide the physical realizations of the de Rham cohomological operators of differential geometry and the discrete symmetry plays the role of Hodge duality operation [18]. It is worthwhile to mention that, in the case of rigid rotor, the components of gauge potential transform in a completely different fashion as compared to other field-theoretic models (see, e.g., [19, 20, 21, 22]). Such study has led us to investigate the present model (i.e. a free particle on a torus) within the framework of superfield approach to BRST formalism.

The contents of our present paper are organized as follows. In the next section, we briefly discuss about the free particle residing on a torus constrained to satisfy a geometrical constraint $(r-a)=0$. We also discuss the Dirac brackets associated with the second-class constrained system. The third section is devoted to the conversion of the second-class constraints into first-class constraints by the introduction of Stückelberg like variables. The local gauge symmetries associated with the first-class constraints are also discussed in this section. The fourth section deals with the derivation of the 
off-shell nilpotent as well as absolutely anticommuting (anti-)BRST transformations within the framework of superfield formalism. In the fifth section, we construct an (anti-)BRST-invariant Lagrangian and derive the conserved (anti-)BRST charges. Finally, in the last section, we make some concluding remarks.

\section{Preliminaries: free particle system on toric geometry and Dirac brackets}

We begin with a free particle of unit mass (i.e. $m=1$ ) forced to satisfy a geometric constraint $(r-a)=0$ on the torus with axial circle in the $x-y$ plane centered at origin of radius $l$, and circular cross-section of radius $r$. The Lagrangian for this constrained system can be written in terms of the toroidal coordinates $(r, \theta, \phi)$ as (see, e.g., [12])

$$
L=\frac{1}{2} \dot{r}^{2}+\frac{1}{2} r^{2} \dot{\theta}^{2}+\frac{1}{2}(l+r \sin \theta)^{2} \dot{\phi}^{2}+\lambda(r-a),
$$

where $\dot{r}=d r / d t, \dot{\theta}=d \theta / d t, \dot{\phi}=d \phi / d t$ are the generalized velocities and $\lambda$ is a Lagrange multiplier. All the variables are function of the time evolution parameter $t$. The coordinates $(r, \theta, \phi)$ for the toric geometry

$$
x=(l+r \sin \theta) \cos \phi, \quad y=(l+r \sin \theta) \sin \phi, \quad z=r \cos \theta,
$$

satisfy the following relation:

$$
r^{2}=\left[\left(x^{2}+y^{2}\right)^{1 / 2}-l\right]^{2}+z^{2}
$$

where the angles $\theta$ and $\phi$ range from 0 to $2 \pi$.

The canonical momenta $p_{\lambda}, p_{r}, p_{\theta}, p_{\phi}$ w.r.t. the dynamical variables $\lambda, r, \theta, \phi$, respectively, are as follows

$$
p_{\lambda}=0, \quad p_{r}=\dot{r}, \quad p_{\theta}=r^{2} \dot{\theta}, \quad p_{\phi}=(l+r \sin \theta)^{2} \dot{\phi} .
$$

In the above, the vanishing momentum $\left(p_{\lambda}=0\right)$ is the primary constraint $\Lambda_{1}=p_{\lambda} \approx 0$ on the theory [23, 24].

By exploiting the Legendre transformations, the canonical Hamiltonian of the system can be written as

$$
H_{c}=\frac{p_{r}^{2}}{2}+\frac{p_{\theta}^{2}}{2 r^{2}}+\frac{p_{\phi}^{2}}{2(l+r \sin \theta)^{2}}-\lambda(r-a) .
$$


In order to find the other constraints, we construct a primary Hamiltonian by adding the primary constraint with an additional Lagrange multiplier $\vartheta$ to the canonical Hamiltonian [23, 24]. Thus, the primary Hamiltonian $H_{p}$ (i.e. $H_{p}=H_{c}+\vartheta p_{\lambda}$ ) takes the following form

$$
H_{p}=\frac{p_{r}^{2}}{2}+\frac{p_{\theta}^{2}}{2 r^{2}}+\frac{p_{\phi}^{2}}{2(l+r \sin \theta)^{2}}-\lambda(r-a)+\vartheta p_{\lambda} .
$$

According to Dirac, constraints must remain intact with time [23]. As a consequence, the time evolution of the primary constraint

$$
\dot{\Lambda}_{1}=\left\{\Lambda_{1}, H_{p}\right\}_{P B}=(r-a) \approx 0,
$$

leads to the secondary constraint on the theory

$$
\Lambda_{2}=(r-a) \approx 0,
$$

where in the computation of the above secondary constraint we have used the following non-vanishing Poisson brackets $\{,\}_{P B}$, namely;

$$
\begin{array}{ll}
\left\{r, p_{r}\right\}_{P B}=1, \quad\left\{\lambda, p_{\lambda}\right\}_{P B}=1, \\
\left\{\theta, p_{\theta}\right\}_{P B}=1, \quad\left\{\phi, p_{\phi}\right\}_{P B}=1 .
\end{array}
$$

Furthermore, the time evolution of the secondary constraint (i.e. $\dot{\Lambda}_{2}=$ $\left\{\Lambda_{2}, H_{p}\right\}_{P B} \approx 0$ ) yields the tertiary constraint

$$
\Lambda_{3}=p_{r} \approx 0
$$

Again, requiring $\Lambda_{3}$ to be time independent (i.e. $\dot{\Lambda}_{3} \approx 0$ ), we have following quaternary constraint

$$
\Lambda_{4}=\frac{p_{\theta}^{2}}{r^{3}}+\frac{p_{\phi}^{2} \sin \theta}{(l+r \sin \theta)^{3}}+\lambda \approx 0 .
$$

Here, we point out that there are no further constraints because the time evolution of the quaternary constraint determines the value of Lagrange multiplier $\vartheta=0$ and the series of constraints terminates here. Thus, we have, in totality, four constraints on the theory, namely;

$$
\begin{aligned}
& \Lambda_{1}=p_{\lambda} \approx 0, \quad \Lambda_{2}=(r-a) \approx 0, \quad \Lambda_{3}=p_{r} \approx 0, \\
& \Lambda_{4}=\frac{p_{\theta}^{2}}{r^{3}}+\frac{p_{\phi}^{2} \sin \theta}{(l+r \sin \theta)^{3}}+\lambda \approx 0 .
\end{aligned}
$$


We note that the above constraints form a set of second-class constraints in the language of Dirac's classification scheme of the constraints 23 .

Now we construct an antisymmetric $4 \times 4$ matrix $C_{i j}$ from the above constraints as follows

$$
C_{i j}=\left\{\Lambda_{i}, \Lambda_{j}\right\}=\left(\begin{array}{cccc}
0 & 0 & 0 & -1 \\
0 & 0 & +1 & 0 \\
0 & -1 & 0 & +\kappa \\
+1 & 0 & -\kappa & 0
\end{array}\right)
$$

where $i, j=1,2,3,4$ and $\kappa=3 p_{\theta}^{2} / r^{4}+3 p_{\phi}^{2} \sin \theta /(l+r \sin \theta)^{4}$. It is evident from (13) that the matrix $C_{i j}$ is a non-singular matrix whose determinant is $(+1)$. The inverse of the above matrix is

$$
C_{i j}^{-1}=\left(\begin{array}{cccc}
0 & +\kappa & 0 & +1 \\
-\kappa & 0 & -1 & 0 \\
0 & +1 & 0 & 0 \\
-1 & 0 & 0 & 0
\end{array}\right)
$$

As a consequence, we can define the Dirac bracket. The Dirac bracket $\{F, G\}_{D}$ between any two dynamical variables $F(q, p)$ and $G(q, p)$ is given by

$$
\{F, G\}_{D}=\{F, G\}_{P B}-\left\{F, \Lambda_{i}\right\}_{P B} C_{i j}^{-1}\left\{\Lambda_{j}, G\right\}_{P B} .
$$

For our second-class system, the Dirac brackets among all the dynamical variables are

$$
\begin{aligned}
& \left\{\theta, p_{\theta}\right\}_{D}=1, \quad\left\{\phi, p_{\phi}\right\}_{D}=1, \quad\{\lambda, \theta\}_{D}=\frac{2 p_{\theta}}{r^{3}}, \\
& \{\lambda, \phi\}_{D}=\frac{2 p_{\phi} \sin \theta}{(l+r \sin \theta)^{3}}, \quad\left\{\lambda, p_{\theta}\right\}_{D}=-\frac{p_{\phi}^{2}(l-2 r \sin \theta) \cos \theta}{(l+r \sin \theta)^{4}} .
\end{aligned}
$$

All the rest of the Dirac brackets turn out to be zero.

To quantize the above constrained system, we promote all the Dirac's Poisson brackets (at the classical level) to the commutators (at the quantum level) and replace all the dynamical variables by their operator form [23, 24].

\section{$3 \quad$ First-class constraints and gauge symmetry}

It is evident from our earlier discussions that the present system is endowed with a set of second-class constraints. To convert the second-class constraints into the first-class constraints, we redefine the variables: $r \rightarrow r-\xi$ and 
$p_{r} \rightarrow p_{r}+p_{\xi}$ (see, e.g., [12]). The canonical Hamiltonian (5) takes the following form:

$$
\tilde{H}_{c}=\frac{\left(p_{r}+p_{\xi}\right)^{2}}{2}+\frac{p_{\theta}^{2}}{2(r-\xi)^{2}}+\frac{p_{\phi}^{2}}{2[l+(r-\xi) \sin \theta]^{2}}-\lambda(r-\xi-a),
$$

and the corresponding first-order Lagrangian is

$$
\begin{aligned}
L_{f} & =\dot{r} p_{r}+\dot{\theta} p_{\theta}+\dot{\phi} p_{\phi}+\dot{\xi} p_{\xi}-\frac{\left(p_{r}+p_{\xi}\right)^{2}}{2}-\frac{p_{\theta}^{2}}{2(r-\xi)^{2}} \\
& -\frac{p_{\phi}^{2}}{2[l+(r-\xi) \sin \theta]^{2}}+\lambda(r-\xi-a) .
\end{aligned}
$$

The above Lagrangian (18) is endowed with the primary constraint $\Omega_{1}=$ $p_{\lambda} \approx 0$ and its time evolution leads to the secondary constraint $\Omega_{2}=(r-$ $\xi-a) \approx 0$. It is to be noted that there are no further constraints because one can show that $\Omega_{2}$ commutes with the canonical Hamiltonian $\tilde{H}_{c}$. Both the constraints (i.e. $\Omega_{1}$ and $\Omega_{2}$ ) are first-class constraints in the Dirac's terminology and this is a signature of the gauge theory.

The most general form of the generator in terms of the first-class constraints, which generates the gauge transformations, can be written as

$$
Q=\dot{\chi} p_{\lambda}-\chi(r-\xi-a)
$$

where $\chi=\chi(t)$ is an infinitesimal and local gauge parameter. The above generator generates the following gauge transformations for all the dynamical variables, namely;

$$
\delta_{(g t)} \lambda=\dot{\chi}, \quad \delta_{(g t)} p_{r}=\chi, \quad \delta_{(g t)} p_{\xi}=-\chi, \quad \delta_{(g t)}\left[r, \theta, \phi, \xi, p_{\theta}, p_{\phi}\right]=0 .
$$

Under the above gauge transformations, the first-order Lagrangian (18) transforms to a total time derivative:

$$
\delta_{(g t)} L_{f}=\frac{d}{d t}[\chi(r-\xi-a)] .
$$

Thus, the action integral (i.e. $S=\int d t L_{f}$ ) remains invariant under the above gauge transformations (20).

\section{Off-shell nilpotent (anti-)BRST symmetries: superfield formalism}

It is clear from our earlier section, the existence of the first-class constraints $\left(\Omega_{1}=p_{\lambda} \approx 0\right.$ and $\left.\Omega_{2}=(r-\xi-a) \approx 0\right)$ implies that the modified theory is a 
gauge theory with the gauge potentials $\lambda, p_{r}$ and $p_{\xi}$. These gauge potentials transform in a completely different way under the gauge transformations (20). As a consequence, we define the exterior derivative $d$ and the 1 -form connection, respectively

$$
\begin{aligned}
d & =d t \partial_{t}+d r \partial_{r}+d \xi \partial_{\xi}, \quad d^{2}=0, \\
A^{(1)} & =d t \lambda(r, \xi, t)+d r B(r, \xi, t)-d \xi E(r, \xi, t),
\end{aligned}
$$

such that our ordinary 3D space is characterized by three coordinates $(r, \xi, t)$. In the superfield formalism [15, 16, we assume that these coordinates are independent variables. We shall see, later on, that the gauge potential components $B(r, \xi, t)$ and $E(r, \xi, t)$ would be connected with $p_{r}$ and $p_{\xi}$, respectively. Finally, at the end of computations, we shall take the limit $(r, \xi) \rightarrow 0$ so that all the dynamical variables of the present theory become only the function of time evolution parameter $t$. Furthermore, we have taken the negative sign in the third term of the 1-form connection $A^{(1)}$ so that the 2-form curvature $d A^{(1)}$ would remain invariant under the gauge and/or (anti-)BRST symmetry transformations. The 2-form curvature is given by

$$
\begin{aligned}
d A^{(1)} & =(d t \wedge d r)\left[\partial_{t} B(r, \xi, t)-\partial_{r} \lambda(r, \xi, t)\right] \\
& -(d t \wedge d \xi)\left[\partial_{t} E(r, \xi, t)+\partial_{\xi} \lambda(r, \xi, t)\right] \\
& -(d r \wedge d \xi)\left[\partial_{r} E(r, \xi, t)+\partial_{\xi} B(r, \xi, t)\right]
\end{aligned}
$$

where we have used the following properties of the wedge products: $d t \wedge d t=$ $0, d r \wedge d r=0, d \xi \wedge d \xi=0, d t \wedge d r=-d r \wedge d t, d t \wedge d \xi=-d \xi \wedge d t$, $d r \wedge d \xi=-d \xi \wedge d r$.

In the superfield formalism, we generalize our $3 \mathrm{D}$ space to $(3,2) \mathrm{D}$ superspace. The $(3,2) \mathrm{D}$ superspace is parametrized, in addition to the ordinary bosonic variables $(r, \xi, t)$, by a pair of Grassmannian variables $(\eta, \bar{\eta})$ (with $\left.\eta^{2}=\bar{\eta}^{2}=0, \eta \bar{\eta}+\bar{\eta} \eta=0\right)$. The exterior derivative $d$ and the 1 -form connection $A^{(1)}$ are also generalized to the super exterior derivative $\tilde{d}$ (with $\tilde{d}^{2}=0$ ) and super 1-form connection $\tilde{A}^{(1)}$ onto $(3,2) \mathrm{D}$ supermanifold as

$$
\begin{aligned}
d \rightarrow \tilde{d} & =d t \partial_{t}+d r \partial_{r}+d \xi \partial_{\xi}+d \eta \partial_{\eta}+d \bar{\eta} \partial_{\bar{\eta}} \\
A^{(1)} \rightarrow \tilde{A}^{(1)} & =d t \tilde{\lambda}(r, \xi, t, \eta, \bar{\eta})+d r \tilde{B}(r, \xi, t, \eta, \bar{\eta})-d \xi \tilde{E}(r, \xi, t, \eta, \bar{\eta}) \\
& +d \eta \bar{F}(r, \xi, t, \eta, \bar{\eta})+d \bar{\eta} F(r, \xi, t, \eta, \bar{\eta})
\end{aligned}
$$

where $\partial_{\eta}=\partial / \partial \eta$ and $\partial_{\bar{\eta}}=\partial / \partial \bar{\eta}$ are the Grassmannian translational generators along $\eta, \bar{\eta}$ directions, respectively. The components of the supervariables can be expanded along the Grassmannian directions as follows

$$
\tilde{\lambda}(r, \xi, t, \eta, \bar{\eta})=\lambda(r, \xi, t)+\eta \bar{f}_{1}(r, \xi, t)+\bar{\eta} f_{1}(r, \xi, t)+i \eta \bar{\eta} B_{1}(r, \xi, t),
$$




$$
\begin{aligned}
\tilde{B}(r, \xi, t, \eta, \bar{\eta}) & =B(r, \xi, t)+\eta \bar{f}_{2}(r, \xi, t)+\bar{\eta} f_{2}(r, \xi, t)+i \eta \bar{\eta} B_{2}(r, \xi, t), \\
\tilde{E}(r, \xi, t, \eta, \bar{\eta}) & =E(r, \xi, t)+\eta \bar{f}_{3}(r, \xi, t)+\bar{\eta} f_{3}(r, \xi, t)+i \eta \bar{\eta} B_{3}(r, \xi, t), \\
F(r, \xi, t, \eta, \bar{\eta}) & =C(r, \xi, t)+i \eta \bar{b}_{1}(r, \xi, t)+i \bar{\eta} b_{1}(r, \xi, t)+i \eta \bar{\eta} s(r, \xi, t), \\
\bar{F}(r, \xi, t, \eta, \bar{\eta}) & =\bar{C}(r, \xi, t)+i \eta \bar{b}_{2}(r, \xi, t)+i \bar{\eta} b_{2}(r, \xi, t)+i \eta \bar{\eta} \bar{s}(r, \xi, t) .
\end{aligned}
$$

Here $b_{1}, \bar{b}_{1}, b_{2}, \bar{b}_{2}, B_{1}, B_{2}, B_{3}$ are the secondary bosonic fields and the secondary fields $f_{1}, \bar{f}_{1}, f_{2}, \bar{f}_{2}, f_{3}, \bar{f}_{3}, s, \bar{s}$ are fermionic in nature. The (anti-) ghost fields $(\bar{C}) C$ (with $C^{2}=\bar{C}^{2}=0, C \bar{C}+\bar{C} C=0$ ) are also fermionic in nature. These secondary (bosonic) fermionic fields can be determined, with the help of horizontality condition ( $\mathrm{HC})$, in terms of the basic and auxiliary fields of the present theory. The following mathematical form of $\mathrm{HC}$

$$
\tilde{d} \tilde{A}^{(1)}=d A^{(1)}
$$

implies that the 1.h.s. has to be independent of the Grassmannian variables $(\eta, \bar{\eta})$ when the $d A^{(1)}$ is generalized onto the $(3,2) \mathrm{D}$ supermanifold. The explicit form of the l.h.s. is

$$
\begin{aligned}
\tilde{d} \tilde{A}^{(1)} & =(d t \wedge d r)\left[\partial_{t} \tilde{B}-\partial_{r} \tilde{\lambda}\right]-(d t \wedge d \xi)\left[\partial_{t} \tilde{E}+\partial_{\xi} \tilde{\lambda}\right] \\
& -(d r \wedge d \xi)\left[\partial_{r} \tilde{E}+\partial_{\xi} \tilde{B}\right]+(d t \wedge d \eta)\left[\partial_{t} \bar{F}-\partial_{\eta} \tilde{\lambda}\right] \\
& +(d t \wedge d \bar{\eta})\left[\partial_{t} F-\partial_{\bar{\eta}} \tilde{\lambda}\right]+(d r \wedge d \eta)\left[\partial_{r} \bar{F}-\partial_{\eta} \tilde{B}\right] \\
& +(d r \wedge d \bar{\eta})\left[\partial_{r} F-\partial_{\bar{\eta}} \tilde{B}\right]+(d \xi \wedge d \eta)\left[\partial_{\xi} \bar{F}+\partial_{\eta} \tilde{E}\right] \\
& +(d \xi \wedge d \bar{\eta})\left[\partial_{\xi} F+\partial_{\bar{\eta}} \tilde{E}\right]-(d \eta \wedge d \bar{\eta})\left[\partial_{\eta} F+\partial_{\bar{\eta}} \bar{F}\right] \\
& -(d \bar{\eta} \wedge d \bar{\eta}) \partial_{\bar{\eta}} F-(d \eta \wedge d \eta) \partial_{\eta} \bar{F} .
\end{aligned}
$$

Exploiting HC, we obtain the following algebraic relationships among the secondary and basic fields, namely;

$$
\begin{aligned}
& b_{1}=0, \quad \bar{b}_{2}=0, \quad s=0, \quad \bar{s}=0, \quad f_{1}=\dot{C}, \quad \bar{f}_{1}=\dot{\bar{C}}, \quad f_{2}=\partial_{r} C, \\
& \bar{f}_{2}=\partial_{r} \bar{C}, \quad \bar{b}_{1}+b_{2}=0, \quad B_{1}=\dot{b}_{2}=-\dot{\bar{b}}_{1}, \quad B_{2}=\partial_{r} b_{2}=-\partial_{r} \bar{b}_{1} \text {, } \\
& f_{3}=-\partial_{\xi} C, \quad \bar{f}_{3}=-\partial_{\xi} \bar{C}, \quad B_{3}=-\partial_{\xi} b_{2}=\partial_{\xi} \bar{b}_{1}, \quad \dot{B}_{3}=-\partial_{\xi} B_{1}, \\
& \partial_{r} B_{3}=-\partial_{\xi} B_{2}, \quad \dot{f}_{2}=\partial_{r} f_{1}, \quad \dot{\bar{f}}=\partial_{r} \bar{f}_{1}, \quad \dot{f}_{3}=-\partial_{\xi} f_{1}, \quad \dot{B}_{2}=\partial_{r} B_{1}, \\
& \dot{\overline{f_{3}}}=-\partial_{\xi} \bar{f}_{1}, \quad \partial_{r} f_{3}=-\partial_{\eta} f_{2}, \quad \partial_{r} \bar{f}_{3}=-\partial_{\xi} \bar{f}_{2} .
\end{aligned}
$$

In the above, we make the choice $b_{2}=-\bar{b}_{1}=b$ and we get the following expressions for the supervariables

$$
\begin{aligned}
\tilde{\lambda}^{(R)}(r, \xi, t, \eta, \bar{\eta}) & =\lambda(r, \xi, t)+\eta \dot{\bar{C}}(r, \xi, t)+\bar{\eta} \dot{C}(r, \xi, t)+i \eta \bar{\eta} \dot{b}(r, \xi, t) \\
\tilde{B}^{(R)}(r, \xi, t, \eta, \bar{\eta}) & =B(r, \xi, t)+\eta \partial_{r} \bar{C}(r, \xi, t)+\bar{\eta} \partial_{r} C(r, \xi, t)
\end{aligned}
$$




$$
\begin{aligned}
& +i \eta \bar{\eta} \partial_{r} b(r, \xi, t), \\
\tilde{E}^{(R)}(r, \xi, t, \eta, \bar{\eta}) & =E(r, \xi, t)-\eta \partial_{\xi} \bar{C}(r, \xi, t)-\bar{\eta} \partial_{\xi} C(r, \xi, t) \\
& -i \eta \bar{\eta} \partial_{\xi} b(r, \xi, t), \\
F^{(R)}(r, \xi, t, \eta, \bar{\eta}) & =C(r, \xi, t)-i \eta b(r, \xi, t), \\
\bar{F}^{(R)}(r, \xi, t, \eta, \bar{\eta}) & =\bar{C}(r, \xi, t)+i \bar{\eta} b(r, \xi, t),
\end{aligned}
$$

where all the variables on the r.h.s. are the functions of $(r, \xi, t)$. The superscript $(R)$ denotes the reduced form of the super expansions.

At this juncture, we make judicious choices for the gauge potentials $B(r, \xi, t)$ and $E(r, \xi, t)$ in terms of the gauge components $p_{r}(r, \xi, t)$ and $p_{\xi}(r, \xi, t)$, respectively, and for their generalizations in the superspace, too. These choices are as follows

$$
\begin{aligned}
B(r, \xi, t) & =\partial_{r} p_{r}(r, \xi, t), & \tilde{B}^{(R)}(r, \xi, t, \eta, \bar{\eta}) & =\partial_{r} \tilde{P}_{r}^{(R)}(r, \xi, t, \eta, \bar{\eta}), \\
E(r, \xi, t) & =\partial_{\xi} p_{\xi}(r, \xi, t), & \tilde{E}^{(R)}(r, \xi, t, \eta, \bar{\eta}) & =\partial_{\xi} \tilde{P}_{\xi}^{(R)}(r, \xi, t, \eta, \bar{\eta}) .
\end{aligned}
$$

Exploiting (29) and (30), we obtain

$$
\begin{aligned}
& \tilde{P}_{r}^{(R)}(r, \xi, t, \eta, \bar{\eta})=p_{r}(r, \xi, t)+\eta \bar{C}(r, \xi, t)+\bar{\eta} C(r, \xi, t)+i \eta \bar{\eta} b(r, \xi, t), \\
& \tilde{P}_{\xi}^{(R)}(r, \xi, t, \eta, \bar{\eta})=p_{\xi}(r, \xi, t)-\eta \bar{C}(r, \xi, t)-\bar{\eta} C(r, \xi, t)-i \eta \bar{\eta} b(r, \xi, t) .
\end{aligned}
$$

Taking the limit $r \rightarrow 0$ and $\xi \rightarrow 0$ in equations (29) and (31), we obtain the physical super expansions on the $(1,2) \mathrm{D}$ supermanifold. These super expansions are

$$
\begin{aligned}
\tilde{\lambda}^{(h)}(t, \eta, \bar{\eta}) & =\lambda(t)+\eta \dot{\bar{C}}(t)+\bar{\eta} \dot{C}(t)+i \eta \bar{\eta} \dot{b}(t) \\
& \equiv \lambda(t)+\eta\left(s_{a b} \lambda\right)+\bar{\eta}\left(s_{b} \lambda\right)+\eta \bar{\eta}\left(s_{b} s_{a b} \lambda\right), \\
\tilde{P}_{r}^{(h)}(t, \eta, \bar{\eta}) & =p_{r}(t)+\eta \bar{C}(t)+\bar{\eta} C(t)+i \eta \bar{\eta} b(t) \\
& \equiv p_{r}(t)+\eta\left(s_{a b} p_{r}\right)+\bar{\eta}\left(s_{b} p_{r}\right)+\eta \bar{\eta}\left(s_{b} s_{a b} p_{r}\right), \\
\tilde{P}_{\xi}^{(h)}(t, \eta, \bar{\eta}) & =p_{\xi}(t)-\eta \bar{C}(t)-\bar{\eta} C(t)-i \eta \bar{\eta} b(t) \\
& \equiv p_{\xi}(t)+\eta\left(s_{a b} p_{\xi}\right)+\bar{\eta}\left(s_{b} p_{\xi}\right)+\eta \bar{\eta}\left(s_{b} s_{a b} p_{\xi}\right), \\
F^{(h)}(t, \eta, \bar{\eta}) & =C(t)-i \eta b(t) \\
\bar{F}^{(h)}(t, \eta, \bar{\eta}) & \equiv C(t)+\eta\left(s_{a b} C\right)+\bar{\eta}\left(s_{b} C\right)+\eta \bar{\eta}\left(s_{b} s_{a b} C\right), \\
& \equiv \bar{C}(t)+i \bar{\eta} b(t)+\eta\left(s_{a b} \bar{C}\right)+\bar{\eta}\left(s_{b} \bar{C}\right)+\eta \bar{\eta}\left(s_{b} s_{a b} \bar{C}\right),
\end{aligned}
$$

where the superscript $(h)$ on the l.h.s. denotes the super expansions obtained after the application of HC.

We point out that the following dynamical variables $r, \theta, p_{\theta}, p_{\phi}$ remain invariant under the gauge transformations [cf. (20)]. Thus, we can apply the 
"augmented" superfield formalism [25, 26, 27, 28] which demands that the gauge-invariant (physical) quantities remain independent of the Grassmannian variables. As a consequence, the supervariables corresponding to the (ordinary) variables $r, \theta, p_{\theta}, p_{\phi}$ remain unaffected by the presence of Grassmannian variables when the latter variables are generalized onto the $(1,2) \mathrm{D}$ supermanifold. Mathematically, this statement can be corroborated as

$$
\begin{aligned}
& \tilde{r}(t, \eta, \bar{\eta})=r(t), \quad \tilde{\xi}(t, \eta, \bar{\eta})=\xi(t), \\
& \tilde{\theta}(t, \eta, \bar{\eta})=\theta(t), \quad \tilde{P}_{\theta}(t, \eta, \bar{\eta})=p_{\theta}(t), \\
& \tilde{\phi}(t, \eta, \bar{\eta})=\phi(t), \quad \tilde{P}_{\phi}(t, \eta, \bar{\eta})=p_{\phi}(t) .
\end{aligned}
$$

From the above super expansions [cf. (32) and (33)], one can easily read out the (anti-)BRST transformations for all the variables. For instance, the BRST transformation $\left(s_{b}\right)$ is equal to the translation of the superfield along $\bar{\eta}$-direction while keeping $\eta$-direction fixed. Similarly, the anti-BRST transformation $\left(s_{a b}\right)$ can be obtained by taking the translation of the superfield along $\eta$-direction and $\bar{\eta}$-direction remains intact. The above statements can be, mathematically, expressed as

$$
\begin{aligned}
s_{b} \Phi(t) & =\left.\frac{\partial}{\partial \bar{\eta}} \tilde{\Phi}(t, \eta, \bar{\eta})\right|_{\eta=0}, \\
s_{a b} \Phi(t) & =\left.\frac{\partial}{\partial \eta} \tilde{\Phi}(t, \eta, \bar{\eta})\right|_{\bar{\eta}=0}, \\
s_{b} s_{a b} \Phi(t) & =\frac{\partial}{\partial \bar{\eta}} \frac{\partial}{\partial \eta} \tilde{\Phi}(t, \eta, \bar{\eta}),
\end{aligned}
$$

where $\tilde{\Phi}(t, \eta, \bar{\eta})$ is the superfield corresponding to the generic dynamical variable $\Phi(t) \equiv r, \theta, \phi, \xi, \lambda, p_{r}, p_{\theta}, p_{\phi}, p_{\xi}, C, \bar{C}$. Exploiting the first two equations in (34), we obtain the following off-shell nilpotent (i.e. $\left.s_{(a) b}^{2}=0\right)$ as well as absolutely anticommuting (i.e. $s_{b} s_{a b}+s_{a b} s_{b}=0$ ) (anti-)BRST symmetry transformations $\left(s_{(a) b}\right)$ :

$$
\begin{aligned}
& s_{a b} \lambda=\dot{\bar{C}}, \quad s_{a b} p_{r}=\bar{C}, \quad s_{a b} p_{\xi}=-\bar{C}, \quad s_{a b} C=-i b, \\
& s_{a b}\left[r, \theta, \phi, \xi, b, \bar{C}, p_{\theta}, p_{\phi}\right], \\
& s_{b} \lambda=\dot{C}, \quad s_{b} p_{r}=C, \quad s_{b} p_{\xi}=-C, \quad s_{b} \bar{C}=i b, \\
& s_{b}\left[r, \theta, \phi, \xi, b, C, p_{\theta}, p_{\phi}\right] .
\end{aligned}
$$

Furthermore, the (anti-)BRST transformations of the auxiliary field $b$ have been derived from the requirements of the nilpotency and/or absolute anticommutativity properties of the (anti-)BRST transformations. We point out 
that, in addition to (35) and (36), the last equation in (34) implies the following non-vanishing transformations: $s_{b} s_{a b} \lambda=i \dot{b}, s_{b} s_{a b} p_{r}=i b, s_{b} s_{a b} p_{\xi}=$ $-i b$.

\section{5 (Anti-)BRST-invariant Lagrangian and con- served charges}

The gauge-fixed Lagrangian which respects the off-shell nilpotent (anti-)BRST symmetry transformations can be written as

$$
\begin{aligned}
L_{b} & =L_{f}-s_{b}\left[i \bar{C}\left(\dot{\lambda}-p_{r}+p_{\xi}+\frac{b}{2}\right)\right] \\
& \equiv L_{f}+s_{a b}\left[i C\left(\dot{\lambda}-p_{r}+p_{\xi}+\frac{b}{2}\right)\right],
\end{aligned}
$$

where $L_{f}$ is our previous Lagrangian [cf. (18)]. The Lagrangian $L_{b}$ in its full blaze of glory can be written as

$$
L_{b}=L_{f}+\frac{b^{2}}{2}+b\left(\dot{\lambda}-p_{r}+p_{\xi}\right)-i \dot{\bar{C}} \dot{C}-2 i \bar{C} C .
$$

Here $b$ is the Nakanishi-Lautrup type auxiliary variable. One can checked that the continuous (anti-)BRST transformations leave the Lagrangian (38) quasi-invariant. To be more precise, under the (anti-)BRST transformations, the Lagrangian transforms to a total time derivative

$$
\begin{aligned}
s_{b} L_{b} & =\frac{d}{d t}[C(r-\xi-a)+b \dot{C}], \\
s_{a b} L_{b} & =\frac{d}{d t}[\bar{C}(r-\xi-a)+b \dot{\bar{C}}] .
\end{aligned}
$$

Thus, the action integral (i.e. $S=\int d t L_{b}$ ) remains invariant under the (anti-)BRST transformations $\left(s_{(a) b}\right)$. According to Noether's theorem, the invariance of the action under the above continuous (anti-)BRST symmetry transformations leads to the following conserved (i.e. $\dot{Q}_{(a) b}$ ) (anti-)BRST charges $Q_{(a) b}$, namely;

$$
Q_{a b}=b \dot{\bar{C}}-\dot{b} \bar{C}, \quad Q_{b}=b \dot{C}-\dot{b} C .
$$

The conservation law $\left(\dot{Q}_{(a) b}=0\right)$ can be proven by exploiting the following Euler-Lagrange equations of motion:

$$
\dot{b}=(r-\xi-a), \quad b=-\left(\dot{\lambda}-p_{r}+p_{\xi}\right), \quad \dot{\theta}=\frac{p_{\theta}}{(r-\xi)^{2}},
$$




$$
\begin{aligned}
& \dot{p}_{r}=\frac{p_{\theta}^{2}}{(r-\xi)^{3}}+\frac{p_{\phi}^{2}}{[l+(r-\xi) \sin \theta]^{3}}-\lambda, \quad \lambda=\dot{p}_{\xi}, \\
& \dot{r}=\left(p_{r}+p_{\xi}\right)+b, \quad \dot{\xi}=\left(p_{r}+p_{\xi}\right)-b, \quad \dot{p}_{\phi}=0, \\
& \dot{\phi}=\frac{p_{\phi}}{[l+(r-\xi) \sin \theta]^{2}}, \quad \dot{p}_{\theta}=\frac{p_{\theta}^{2}(r-\xi) \cos \theta}{[l+(r-\xi) \sin \theta]^{3}}, \\
& \ddot{\bar{C}}-2 \bar{C}=0, \quad \ddot{C}-2 C=0 .
\end{aligned}
$$

The above equations of motion have been derived from the Lagrangian (38).

We point out that the nilpotency (i.e. $Q_{(a) b}^{2}=0$ ) and the anticommutativity (i.e. $Q_{b} Q_{a b}+Q_{a b} Q_{b}=0$ ) of the (anti-)BRST charges $Q_{(a) b}$ can be proven in a simple and straightforward manner

$$
\begin{aligned}
s_{b} Q_{b} & =-i\left\{Q_{b}, Q_{b}\right\}=0 \Rightarrow Q_{b}^{2}=0, \\
s_{a b} Q_{a b} & =-i\left\{Q_{a b}, Q_{a b}\right\}=0 \Rightarrow Q_{a b}^{2}=0, \\
s_{b} Q_{a b} & =-i\left\{Q_{a b}, Q_{b}\right\}=0 \Rightarrow Q_{b} Q_{a b}+Q_{a b} Q_{b}=0, \\
s_{a b} Q_{b} & =-i\left\{Q_{b}, Q_{a b}\right\}=0 \Rightarrow Q_{b} Q_{a b}+Q_{a b} Q_{b}=0 .
\end{aligned}
$$

In the above, the first and second lines show the nilpotency of the BRST and anti-BRST charges, respectively whereas the third and fourth lines imply the absolute anticommutativity of the (anti-)BRST charges.

Before we close this section, we lay emphasis on the fact that the physicality condition $Q_{(a) b}|p h y s\rangle=0$, on the conserved (anti-)BRST charges $Q_{(a) b}$, yields

$$
\begin{aligned}
& b \mid \text { phys }\rangle=0 \Rightarrow p_{\lambda}|p h y s\rangle=0 \\
& \dot{b} \mid \text { phys }\rangle=0 \Rightarrow(r-\xi-a)|p h y s\rangle=0
\end{aligned}
$$

where $p_{\lambda}=\partial L_{b} / \partial \dot{\lambda}=b$ is the canonical momentum w.r.t the dynamical variable $\lambda$. These conditions imply that the operator form of the first-class constraints $\Omega_{1}=p_{\lambda} \approx 0, \Omega_{2}=(r-\xi-a) \approx 0$ annihilates the physical state $(|p h y s\rangle)$ of the total quantum Hilbert space of states. As a result, the above physicality criteria $Q_{(a) b}|p h y s\rangle=0$ are consistent with the Dirac's quantization of the constrained system [23, 24].

\section{Conclusions}

In our present investigation, we have studied a free particle residing on a torus constrained to satisfy the geometric constraint $(r-a)=0$. This model is endowed with a set of four second-class constraints in the Dirac terminology. Thus, we have derived the Dirac brackets. Furthermore, by incorporating the 
Stückelberg like variables $\left(\xi, p_{\xi}\right)$, the second-class constraints turn into the first-class constraints. As a result, the modified theory respects the gauge symmetries. These gauge transformations have been derived from the firstclass constraints.

We lay emphasis on the fact that the components of gauge potentials (i.e. $\left.\lambda, p_{r}, p_{\xi}\right)$ transform quite differently under the gauge transformations (20). Thus, the derivation of the proper (anti-)BRST transformations was not straightforward. However, a similar kind of work has been carried out for the model of rigid rotor 18 where a non-trivial choice for the gauge potential has been made which is quite different from the other gauge field theoretic models (see, e.g., [19, 20]). This study has led us to construct a 1-form connection [cf. (22)] and we have made some judicious choices for the (super) gauge potentials [cf. (30)]. Within the framework of superfield formalism, we have incorporated the gauge components $p_{r}$ and $p_{\xi}$ very carefully [cf. (30)]. Exploiting the celebrated $\mathrm{HC}$ and taking the limits $r \rightarrow 0, \xi \rightarrow 0$ (at the end of computations) in the super expansions (29) and (31), we obtain the proper off-shell nilpotent as well as absolutely anticommuting (anti-)BRST transformation [cf. (35), and (36)].

It is to be noted that the variable $\lambda$ is no longer a Lagrange multiplier within the framework of BRST formalism. As it can be checked, the (anti-) BRST-invariant Lagrangian (38) contains a time derivative of $\lambda$. Thus, it is a dynamical variable. The continuous (anti-)BRST symmetry transformations lead to the conserved, nilpotent and anticommuting (anti-)BRST charges. Furthermore, the physicality criteria $Q_{(a) b}|p h y s\rangle=0$ produce the first-class constraints $p_{\lambda} \approx 0,(r-\xi-a) \approx 0$ [cf. (43)]. Thus, the physicality criteria $Q_{(a) b} \mid$ phys $\rangle=0$ establish the connection between the BRST quantization and the Dirac's quantization of constrained system [23, 24].

We point out that our present approach can also be generalized for the relativistic field-theoretic models (see, e.g., [21, 29]) where the celebrated $\mathrm{HC}$ and gauge-invariant restriction(s) play an important role to derive the nilpotent (anti-)BRST symmetry transformations within the framework of superfield formalism. It would be a nice work to look for the generalization of the present approach for the higher-form and higher dimensional (non-) Abelian gauge field theories. It is interesting to point out that, within the framework of BRST formalism, the present model would turn out to be a model for the Hodge theory where all the de Rham cohomological operators of differential geometry finds their physical realizations in terms of the symmetry properties [18, 19, 20, 21, 22, 29]. We shall investigate this issue in our future work [30]. 


\section{References}

[1] Green M. B., Schwarz, J. H. and Witten E., Superstring Theory (Cambridge University Press, Cambridge, 1987).

[2] Polchinski J., String Theory (Cambridge University Press, Cambridge, 1987).

[3] Chaichian M., Demechev A., Presnajder P., Sheikh-Jaabbari M. M., and Turreanu A., Nucl. Phys. B 611, 383 (2001).

[4] Morariu B. and Polychronakos A., Nucl. Phys. B 610, 531 (2001).

[5] Namsrai Kh. Torus Theory, ICTP Preprints, Report No.: IC/IR/2001/153 (2001).

[6] Namsrai Kh. Square-root quantization of elementary particle masses and charges, ICTP Preprints, Report No.: IC/IR/2001/18 (2001).

[7] Namsrai Kh. and Geramb H.V. von., Int. J. Theor. Phys. 40, 1929 (2001).

[8] Becchi C., Rouet A. and Stora R., Phys. Lett. B 52, 344 (1974).

[9] Becchi C., Rouet A. and Stora R., Commun. Math. Phys. 42, 127 (1975).

[10] Becchi C., Rouet A. and Stora R., Ann. Phys. (N. Y.) 98, 287 (1976).

[11] Tyutin I. V., Lebedev Institute Preprint, Report No. FIAN-39 (1975) (Unpublished).

[12] Hong S.-T., Mod. Phys. Lett. A 20, 1577 (2005).

[13] Fradkin E. S. and Vilkoviski G. A., Phys. Lett. B 55, 224 (1975).

[14] Henneaux M., Phys. Rep. 126, 1 (1985).

[15] Bonora L. and Tonin M., Phys. Lett. B 98, 48 (1981).

[16] Bonora L., Pasti P. and Tonin M., Nouvo Cimento A 63, 353 (1981).

[17] Nemeschansky D., Preitschopf C. and Weinstein M., Ann. Phys. (N. Y.) 183, 226 (1988).

[18] Gupta S. and Malik R. P., Eur. Phys. J. C 68, 325 (2010).

[19] Malik R. P., Int. J. Mod. Phys. A 22, 3521 (2007). 
[20] Gupta S. and Malik R. P., Eur. Phys. J. C 58, 517 (2008).

[21] Bhanja T., Shukla D. and Malik, R. P., Eur. Phys. J. C 73, 2535 (2013).

[22] Kumar R., Krishna S, Shulka A. and Malik R. P., Eur. Phys. J. C 72, 1980 (2012).

[23] Dirac P. A. M., Lectures on Quantum Mechanics, Belfer Graduate School of Science (Yeshiva University Press, New York, 1964).

[24] Sundermeyer K., Constrained Dynamics. Lecture Notes in Physics, 169 (Springer, Berlin, 1982).

[25] Malik, R. P., Phys. Lett. B 584, 210 (2004).

[26] Malik R. P., J. Phys. A: Math. Gen. 37, 5261 (2004).

[27] Malik, R. P., Eur. Phys. J. C 51, 169 (2007).

[28] Gupta S., Kumar R. and Malik R. P. (To appear in Can. J. Phys., 2014).

[29] Gupta, S., Mod. Phys. Lett. A 15, 1450076 (2014).

[30] Kumar. R., in preparation. 